\title{
Effect of cross-sectional area reduction rate and alloy composition on the formation of $<001>$-fiber texture in Ti-Mo-Al-Zr alloy wire
}

\author{
Yuri Shinohara $^{* 1,2}$, Yoshiki matsumoto ${ }^{3}$, Masaki Tahara ${ }^{1,2}$, Hideki Hosoda ${ }^{1,2}$ and Tomonari Inamura ${ }^{1,2}$ \\ ${ }^{1}$ Laboratory for Materials and Structures, Institute of Innovative Research, Tokyo Institute of Technology, 4259, Nagatsutacho, Midori-ku, Yokohama 226-8503, Japan \\ ${ }^{2}$ Laboratory for Future Interdisciplinary Research of Science and Technology, Institute of Innovative Research, Tokyo Institute of Technology, 4259, Nagatsutacho,
}

Midori-ku, Yokohama 226-8503, Japan

${ }^{3}$ Tokyo Institute of Technology, 4259, Nagatsutacho, Midori-ku, Yokohama 226-8503, Japan

Corresponding author E-mail: shinohara.y.aa@m.titech.ac.jp

\begin{abstract}
Texture formation in Ti-7Mo-8Al-6Zr cold-groove-rolled alloy wires was investigated and compared to that of Ti-5.5Mo-8Al-6Zr. Irregularly textured $<001>$-fiber was formed in Ti-5.5Mo-8Al-6Zr. The Ti-7Mo-8Al-6Zr cold-groove-rolled wire mainly composed of the $\beta$ phase though $\alpha^{\prime \prime}$ martensite was induced by cold-groove-rolling in the Ti-5.5Mo-8Al-6Zr alloy. From the TEM observations of the severely deformed cold-rolled sheet, $\alpha^{\prime \prime}$ martensite was assumed to be induced during cold-groove-rolling and trace $\alpha^{\prime \prime}$ was assumed to be retained after cold-groove-rolling. This texture is common in bcc metals and corresponds to the $\langle 010\rangle{ }^{\prime \prime}$-fiber which is formed in the Ti-5.5Mo$8 \mathrm{Al}-6 \mathrm{Zr}$ alloy when the lattice correspondence between $\beta$ and $\alpha^{\prime \prime}$ is considered. On the other hand, the $<001>$-fiber was strongly developed in the cold-groove-rolled wire with a reduced cross sectional area of $98 \%$ following solution treatment, while this texture was not observed in the specimen with a $60 \%$ reduced cross sectional area. The texture formed in solution-treated Ti-7Mo-8Al-6Zr alloy wire was similar to that of the Ti-5.5Mo-8Al-6Zr alloy, although the dominant phase in the cold-groove-rolled specimen differed.
\end{abstract}

\section{Introduction}

$\beta$-Ti shape memory alloys (SMAs) have attracted significant attention as implant materials because of their excellent biocompatibility and corrosion resistance [1, 2]. A low Young's modulus $(\sim 30 \mathrm{GPa})$ is required for implant materials because differences in the Young's modulus between the bone and implant can cause bone resorption [2]. The Young's modulus of $\beta$-Ti is lower than that of other alloys, but it is still higher than required for implants: $50 \mathrm{GPa}$ for Ti-Nb-Ta-Zr (TNTZ)[3, 4] and 200 GPa for SUS316L[1].

It has been reported that texture formation affects the Young's modulus along the rolling direction (RD) in polycrystalline materials. Hence, reduction in Young's modulus can be achieved through texture control. The $<110>/ / \mathrm{RD}$ textures are mainly formed as deformation or recrystallization textures: $\{001\}<110>,\{111\}<110>,\{112\}<110>$, and $\{111\}<112>$ for sheets, and $<110>$ - and $<102>$-fibers for wire [5-8]. Indices without a subscript and with $\alpha^{\prime \prime}$ refer to $\beta$ and martensite phases, respectively. However, the Young's modulus of $\beta$-Ti alloy along the $<110>$ direction is higher than the average Young's modulus for a randomly oriented polycrystal, which is unsuitable for Young's modulus reduction $[9,10]$.

Recently, irregular texture formation, $<001>/ / \mathrm{RD}$, was reported in a Ti-5.5Mo-8Al-6Zr (mol\%; hereafter 5.5Mo alloy) alloy sheet and groove-rolled wire material [11]. Goss-oriented $(\{110\}<001>$ ) and $<001>$-fibers are dominantly formed as a recrystallization texture in sheet and cold-groove-rolled (hereafter, simply groove-rolled) wire materials, respectively. In $\beta$-Ti, the Young's modulus is lowest along $\langle 001\rangle[10,12]$ and the development of this orientation is effective to reduce the Young's modulus in both sheet and wire materials $[11,13]$. Particularly for wire materials, it was observed that: (1) stress-induced martensitic transformation (SIMT) from $\beta$ (bcc) to $\alpha^{\prime \prime}$ (corthorhombic) occurs during groove-rolling and (2) the $<001>$-fiber developed by severe deformation after heat treatment. However, little information is available regarding the alloy composition. The formation of $\alpha^{\prime \prime}$ during deformation is considered to be important for $<001>$-fiber formation. Thus, understanding the relationship between alloy composition and texture formation in Ti-Mo-Al-Zr alloys is important as the alloy composition affects the martensitic transformation temperature and stress for inducing martensite $[14,15]$. Hence, texture formation in Ti-7Mo-8Al-6Zr groove-rolled wire with higher Mo content compared to conventional Ti-5.5Mo-8Al-6Zr was investigated. Effect of cross-sectional area reduction on the texture formation was also evaluated.

\section{Material and experiments}

First, a Ti-7Mo-8Al-6Zr (mol\%) alloy was fabricated by Ar-arc melting in an Ar- $1 \% \mathrm{H}_{2}$ atmosphere and homogenized at $1273 \mathrm{~K}$ for $7.2 \mathrm{ks}$. The obtained ingot was grooverolled with nominal reductions in the cross sectional area (CSA, $r$ ) of $60 \%$. Subsequently, a part of the wire was annealed at $1173 \mathrm{~K}$ for $1.8 \mathrm{ks}$ and extra-groove-rolled to $r=$ $98 \%$. The specimens were sliced from the wires and solution-treated at $1173 \mathrm{~K}$ for $3.6 \mathrm{ks}$. Cross-sections along the length (lengthwise-sliced specimens) and diameter (roundsliced specimens) of the wire were prepared. The specimens were named based on their $r$ value and thermomechanical process. For example, "GR98" denotes the grooverolled specimen with $r=98 \%$ and "ST98" denotes the solution-treated specimen of "GR98". All heat-treatment processes (homogenization, intermediate annealing, and solution treatment) were performed under an $\mathrm{Ar}$ atmosphere and heat-treated samples were quenched in water.

The phase constituents of the lengthwise-sliced specimens were identified via $\theta-2 \theta$ X-ray diffractometry (XRD). The textures of the round-sliced specimens were evaluated using X-ray pole figure (XPF) measurements obtained on an X-ray diffractometer with a polycarpellary X-ray lens and parallel plate collimator. The elevation angle was varied from 0 to $75^{\circ}$. The specimens for these measurements were prepared by electropolishing in a mixture of $6 \mathrm{vol} \%$ perchloric acid, $35 \mathrm{vol} \%$ butanol, and $59 \mathrm{vol} \%$ methanol at $233 \mathrm{~K}$. For further details of the sample preparation, see Ref. [11].

A cold-rolled sheet with a reduced thickness of $98 \%$ (hereafter, CR98) was also prepared for microstructure observation of a severely deformed sample. Transmission electron microscopy (TEM) was performed and the observed specimens were finished using a twin-jet polishing technique in a mixture of $2 \mathrm{vol} \%$ hydrofluoric acid, $5 \mathrm{vol} \%$ sulfuric acid, and $93 \mathrm{vol} \%$ methanol at $233 \mathrm{~K}$

(C) The Authors, published by EDP Sciences. This is an open access article distributed under the terms of the Creative Commons Attribution License 4.0 (http://creativecommons.org/licenses/by/4.0/). 


\section{Results and discussion}

Figure 1 shows the obtained $\theta-2 \theta$ XRD profiles of the prepared samples. Only peaks originating from the $\beta$ phase were confirmed in all specimens (GR60, GR98, CR98, ST60, and ST98). The phase constituents in the GR and CR specimens differed from those of the 5.5Mo alloy. SIMT was observed and martensite remained after cold- or groove-rolling of the $5.5 \mathrm{Mo}$ alloy $[11,16]$. The $\beta$ phase in the studied alloy was stabilized to a greater extent than in the $5.5 \mathrm{Mo}$ alloy because Mo is the $\beta$ stabilizer for $\beta$-Ti alloys $[14,15,17]$. Thus, the martensitic transformation suppression during groove- or cold-rolling and/or reverse transformation after groove- or cold-rolling at room temperature occurred. Peaks in the spectra of the ST specimens were sharper than those observed in the spectra of the GR or CR specimens. The different peak shapes imply dislocation introduction by groove- or cold-rolling and recrystallization or recovery upon solution treatment.

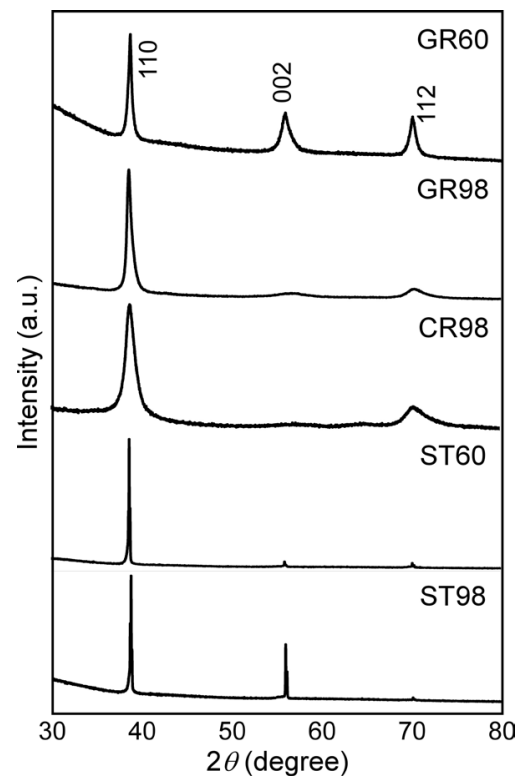

Fig. 1 XRD profiles of the Ti-7Mo-8Al-6Zr alloy.

Figure 2 shows the 110 XPFs of the GR specimens where the $<110>$-fiber was formed in both GR60 and GR98. This texture is common in bcc metals and corresponds to the $<010\rangle_{\alpha^{\prime \prime}}$-fiber which was formed in 5.5Mo alloy when the lattice correspondence between $\beta$ and $\alpha^{\prime \prime}$ is considered. The 001 XPFs of the ST specimens are shown in Fig. 3 where the $<001>$-fiber developed dramatically in ST98, but was not observed to a significant degree in ST60. This is consistent with the $<001>$-fiber formation in Ti-5.5Mo8Al-6Zr alloy, i.e., the texture develops with increasing $r$.

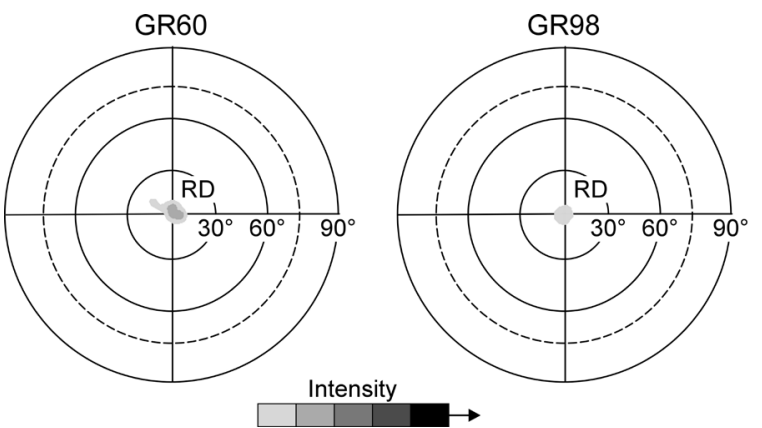

Fig. 2110 PFs for the GR60 and GR98 samples. The broken line indicates the measurement range limit.

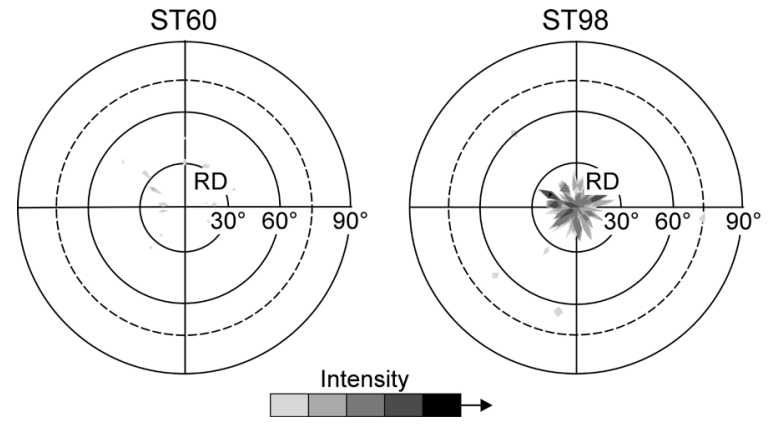

Fig. 3002 PFs for the ST60 and ST98 samples. The broken line indicates measurement range limit.

The texture formed in the severe groove-rolled specimen after solution treatment of the 7Mo alloy was the same as that of the 5.5Mo alloy, although the dominant phase in the GR specimen was different. The microstructure in the severely deformed 7Mo alloy was observed in detail. Figure 4 shows the TEM images of the CR98 specimen. Fine microstructure and Debye-Scherrer ring-like patterns were observed. A weak diffraction ring was observed in the vicinity of the 211 ring, as indicated by the white arrow. Hence, the intensity profile as a function of the distance from the transmission spot in reciprocal space $(d)$ was determined. Figure 5 shows the intensity profile along the broken line indicated in Fig. 4(b). The peaks at $1 / d=0.23,0.16$ and $0.13 \mathrm{~nm}$ were indexed as 110,002 and 211 , respectively. In addition, weak peaks were observed at $1 / d=$ 0.25 and $0.14 \mathrm{~nm}$, indexed as $002^{\prime \prime}$ and $131_{\alpha^{\prime \prime}}$. This indicates that SIMT occurred in the CR98 specimen of the 7Mo alloy. The volume fraction of $\alpha^{\prime \prime}$ was considered to be so small that it could not be detected in the $\theta-2 \theta$ XRD results (Fig. 1). It is reasonable to suppose that SIMT occurred and trace $\alpha^{\prime \prime}$ was present in the GR98 specimen even though the rolling method was different. If we assume that $\alpha^{\prime \prime}$ formed by groove-rolling is essential for $<001>$-fiber formation, we can propose the following mechanism: (1) SIMT occurs during groove-rolling, (2) irregular slip systems are activated and deformation texture is formed, (3) most $\alpha^{\prime \prime}$ martensite undergo a reverse transformation to $\beta$ 
after groove-rolling, and (4) the $<001>_{\beta}$-fiber forms as a recrystallization texture after solution treatment. However further investigation regarding the role of $\alpha^{\prime \prime}$ formation in the development of $<001>$-fiber is required because the rolling texture in the present alloy is common in conventional $\beta$-Ti alloys.
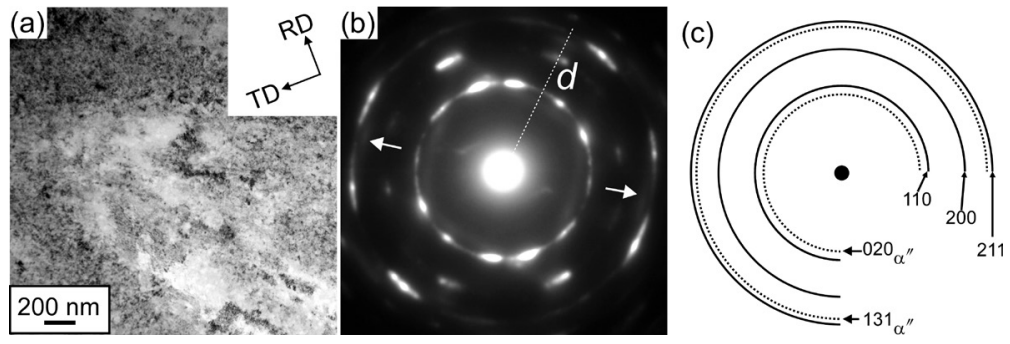

Fig. 4 (a) A bright-field image and selected area diffraction pattern (SADP) of the CR98 sample. (c) Key diagram of the SADP. The solid and broken lines in (c) indicate reflections from the $\beta$ and $\alpha^{\prime \prime}$ phases, respectively.

\section{Plane spacing, $\mathrm{nm}$}

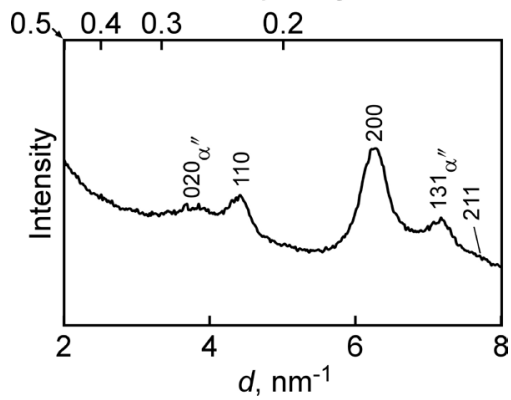

Fig. 5 Intensity profile along the white broken line in Fig. 4 (b).

\section{Conclusion}

(1) The $\theta-2 \theta$ XRD measurements revealed that the Ti-7Mo-8Al-6Zr cold-groove-rolled wire was mainly composed of the $\beta$ phase. From the TEM observations of the severely deformed cold-rolled sheet, $\alpha^{\prime \prime}$ martensite was assumed to be induced during cold-groove-rolling and trace $\alpha^{\prime \prime}$ was assumed to be retained after cold-groove-rolling.

(2) The $<110>$-fiber was developed in the cold-groove-rolled wire specimens. This texture is common in bcc metals and corresponds to the $<010>\alpha^{\prime \prime}-$ fiber which is formed in $5.5 \mathrm{Mo}$ alloy when a mixed $\beta$ and $\alpha^{\prime \prime}$ lattice is considered.

(3) The $<001>$-fiber was developed in the cold-groove-rolled wire with a $98 \%$ reduced cross sectional area followed by solution treatment.

\section{Acknowledgements}

This work was supported by the Japan Society for the Promotion of Science (Grant numbers Wakate B: 17K14833, Wakate A: 17H04959, Kiban S: 26220907, and Kiban B: 18H01728).

\section{References}

[1] M. Niinomi: Metall. Mater. Trans. A 33 (2002) 477-486.

[2] M. Niinomi: Sci. Technol. Adv. Mater. 4 (2003) 445-454.

[3] M. Nakai, M. Niinomi, T. Oneda: Metall. Mater. Trans. A 43 (2011) 294-302.

[4] D. Kuroda, M. Niinomi, M. Morinaga, Y. Kato, T. Yashiro: Mater. Sci. Eng., A 243 (1998) 244-249.

[5] S. Cai, M.R. Daymond, Y. Ren, D.M. Bailey, L.E. Kay: Mater. Sci. Eng., A 562 (2013) 172-179.

[6] S. Cai, Y. Ren, L.E. Kay: Scripta Mater. 68 (2013) 518-521.

[7] I. Kubota, T. Inamura, K. Wakashima, H. Hosoda: SMST-2007 (2008) 533-536.

[8] S. Hanada, N. Masahashi, T.K. Jung, M. Miyake, Y.S. Sato, H. Kokawa: J. Mech. Behav. Biomed. Mater. 32 (2014) $310-320$.

[9] H.Y. Kim, T. Sasaki, K. Okutsu, J.I. Kim, T. Inamura, H. Hosoda, S. Miyazaki: Acta Mater. 54 (2006) 423-433. 
[10] T. Inamura, H. Hosoda, K. Wakashima, S. Miyazaki: Mater. Trans. 46 (2005) 1597-1603.

[11] Y. Shinohara, Y. Matsumoto, M. Tahara, H. Hosoda, T. Inamura: Materialia 1 (2018) 52-61.

[12] M. Tane, S. Akita, T. Nakano, K. Hagihara, Y. Umakoshi, M. Niinomi, H. Nakajima: Acta Mater. 56 (2008) 2856-2863.

[13] Y. Shinohara, D. Narita, M. Tahara, H. Hosoda, T. Inamura: Mater. Trans. 57 (2016) 1998-2001.

[14] A.V. Dobromyslov, V.A. Elkin: Scripta Mater. 44 (2001) 905-910.

[15] Y. Al-Zain, H.Y. Kim, H. Hosoda, T.H. Nam, S. Miyazaki: Acta Mater. 58 (2010) 4212-4223.

[16] T. Sasaki, K. Hiramatsu, M. Tahara, H. Hosoda, S. Miyazaki, T. Inamura: Adv. Mat. Res. 922 (2014) 622-625.

[17] H.Y. Kim, Y. Ohmatsu, J.I. Kim, H. Hosoda, S. Miyazaki: Mater. Trans. 45 (2004) 1090-1095. 